Pacific Journal of Mathematics

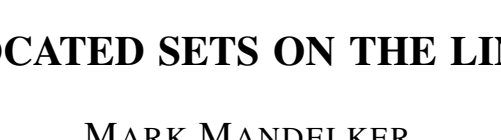




\section{LOCATED SETS ON THE LINE}

\section{MARK MANDELKERN}

Located sets are sets from which the distance of any point may be measured; they are used extensively in modern constructive analysis. Here a general method is given for the construction of all located sets on the line. It is based on a characterization of a located set in terms of the resolution of its metric complement into a union of disjoint open intervals. The characterization depends on a strong countability condition for the intervals, called the locating condition. Included as a special case is the characterization and construction of compact sets. The techniques used are in accord with the principles of Bishop's Foundations of Constructive Analysis, 1967.

In many situations it is desired to measure the distance

$$
\rho(x, G) \equiv \inf \{\rho(x, y): y \in G\}
$$

between a point $x$ and a set $G$ in a metric space. However, this is not always possible constructively. By this we mean that a counterexample exists in the sense of Brouwer; discussions of these are found in [1] (and [4]). The italicized word "not" will also be used below in this sense.

Brouwer [2] introduced the concept of located set, for which the above distances always exist. Here the concept of located set on the line is reduced to the concept of number. The construction of an arbitrary located set is reduced to the construction of two sequences of real numbers with certain properties.

The metric complement of a located set $G$ is the set

$$
-G \equiv\{x: \rho(x, G)>0\} .
$$

Such a set is said to be colocated.

The characterization of a located set $G$ on the line is obtained by means of the resolution of its metric complement $-G$ into a countable union $\bigcup_{n} I_{n}$ of disjoint open intervals, given in [4]. It is shown in [3] that only the closure of a located set $G$ may be recovered from its metric complement $-G$. Thus we characterize closed located sets. Arbitrary located sets are precisely the dense subsets of these.

The characterization theorem below involves four conditions on the sequence $\left\{I_{n}\right\}$ of open intervals. Briefly, these are the following.

(1) The intervals are fixative, i.e., each is either void or fixed 
(contains some point).

(2) The intervals are disjoint.

(3) The locating condition. This limits the number of intervals of a given size which intersect a given bounded segment, and locates them in the sequence of intervals.

(4) The representation of $G$ as an intersection of notched lines (i.e., lines with an interval removed), complementary to the resolution of $-G$ as a union of intervals.

The essential property of located sets is expressed in the locating condition (3). For any point $x$ on the line and for any $\varepsilon>0$, this condition specifically locates the finitely many intervals in the sequence $\left\{I_{n}\right\}$ which might contain $x$ and have length more than $\varepsilon$.

The set $G$ is formed by notching out from the line the succession of open intervals. From the point of view of a given point $x$, the distance $\rho(x, G)$ seems to be 0 during this process, until, perhaps, $x$ is notched out. When the notch $I_{n}$ is taken, any finite endpoint of this interval is assured a permanent place in $G$, because under the disjointness condition no succeeding notch can remove it. Thus the exact distance is known as soon as $x$ is notched out; however, whether this happens might not be predictable. Therefore, an estimate of the distance requires a prediction giving the specific location of those intervals which might remove $x$ and also be large enough to produce a large distance. Thus, if finitely many intervals have been considered, and a prediction is given that the remaining intervals either stay clear of $x$ or are of length less than $\varepsilon$, then $\rho(x, G)$ may be calculated to within $\varepsilon$.

It is easily concluded that only finitely many disjoint intervals of length greater than $1 / k$ can meet $(-k, k)$, by considering their total length. However, such methods will not suffice here, for their location in the entire sequence $\left\{I_{n}\right\}$ of intervals is not determined. The locating condition (3) actually lists a finite number $M_{k}$ of intervals which includes all those in question.

To construct a closed located set $G$ on the line using the characterization below, proceed as follows.

I. First choose any finite number $M_{1}$ of open intervals $I_{n} \equiv$ $\left(a_{n}, b_{n}\right)$ of any length, satisfying (1) and (2). To satisfy the fixative condition (1), decide whether $\left(a_{n}, b_{n}\right)$ is to be void or fixed, and specify $(1,0)$, or ensure $a_{n}<b_{n}$, accordingly. To satisfy the disjointness condition (2), after $m$ intervals have been chosen use step (x) in the proof to examine the finitely many segments, in one of which the next interval must be placed.

II. Next choose any finite number $m_{2}$ of open intervals in the same way, and also, to satisfy the locating condition (3), ensure 
that any of these $m_{2}$ intervals which meet $(-1,1)$ are of length $\leqq 1$. Put $M_{2} \equiv M_{1}+m_{2}$.

III. Continue in this way. For example, any of the next $m_{3}$ intervals which meet $(-2,2)$ must be of length $\leqq 1 / 2$.

IV. The set $G$ obtained is the set of all points $x$ such that, for each $n$, either $x \leqq a_{n}$ or $x \geqq b_{n}$.

An open interval is a set of the form $(a, b) \equiv\{x \in R: a<x<b\}$. To include the unbounded intervals, $a$ and $b$ are taken to be extended real numbers. To include also the case of an open interval for which it may be unknown whether it is bounded or unbounded, we use the system $R^{\infty}$ of extended real numbers constructed in $[4, \S 4]$. Thus it may be unknown whether an endpoint is finite or infinite.

Allowing $+\infty$ as a distance, we consider the void set to be located. Furthermore, the extended real numbers in $R^{\infty}$ will be used as distances. Thus it may be unknown whether a given located set $G$ is void or fixed, since it may be unknown whether $\rho(x, G)$ is infinite or finite. For example, if $\left\{a_{n}\right\}$ is an increasing sequence of zeros and ones for which it is unknown whether or not some $a_{n}=1$, then the set $\left\{n: a_{n}=1\right\}$ is not fixative; yet it is located in the sense of distances measured with extended real numbers. Although the distances $\rho(x, G)$ used in the study of located sets $G$ are extended real numbers, the points of $G$ and the points $x$ considered are always finite real numbers.

A set on the line is compact if and only if it is closed, located, bounded, and fixed. Thus, compact sets are characterized by the conditions of the theorem together with the following additional conditions:

(5) (bounded) Some $a_{n}=-\infty$ and some $b_{k}=+\infty$.

(6) (fixed) For each $n$, either $a_{n}>-\infty$ or $b_{n}<+\infty$. (See [5, Theorem 3].)

An example in which the locating condition (3) does not hold, and thus where the set $G$ defined by (4) is not located, is easily derived from [3, Example 2]. The question raised by that example, of when a countable union of fixative disjoint open intervals is colocated, is answered by the locating condition in the corollary below.

Only constructive properties of the real numbers and extended real numbers, such as are found in [1] and [4], will be admitted. We shall need to perform certain limited algebraic operations in $R^{\infty}$. If $s \equiv\left\{s_{n}\right\}$ is an extended real number (where $\left\{s_{n}\right\}$ is an extended Cauchy sequence of real numbers), and $a$ is a finite number, then $s \pm a$ and $a-s$ are easily defined in $R^{\infty}$; for example, $s+a \equiv$ $\left\{s_{n}+a\right\}$. Furthermore, although in general extended real numbers 
$a$ and $b$ can not be subtracted, if $a<b$ then $b-a$ is easily defined. Thus we define the length $l(I) \equiv b-a$ of any fixed open interval $I \equiv(a, b)$.

THEOREM. For any closed located set $G$ on the line, there exist sequences $\left\{a_{n}\right\}$ and $\left\{b_{n}\right\}$ of extended real numbers such that

(1) $a_{n} \neq b_{n}$, for all $n$.

(2) $b_{n} \leqq a_{k}$ or $b_{k} \leqq a_{n}$, whenever $n \neq k$ and $a_{n}<b_{n}$ and $a_{k}<b_{k}$.

(3) There exists a sequence $\left\{M_{k}\right\}$ of positive integers such that $n \leqq M_{k}$ whenever $\left(a_{n}, b_{n}\right)$ meets $(-k, k)$ and $b_{n}-a_{n}>1 / k$.

(4) $G=\bigcap_{n}\left(\left(-\infty, a_{n}\right] \cup\left[b_{n},+\infty\right)\right)$.

Conversely, whenever $\left\{a_{n}\right\}$ and $\left\{b_{n}\right\}$ are sequences of extended real numbers satisfying (1), (2) and (3), then the set $G$ defined by (4) is closed and located.

Proof. Necessity. Let $G$ be a closed located set.

(i) Definitions. Let

$$
-G=\bigcup_{n}\left(a_{n}, b_{n}\right)
$$

be the resolution of the colocated set $-G$ into a countable union of fixative disjoint open intervals, as obtained in [4]. The intervals $\left(a_{n}, b_{n}\right)$ are called notches. When the notch $\left(a_{n}, b_{n}\right)$ is fixed, the numbers $a_{n}$ and $b_{n}$ are called endpoints. Clearly, all finite endpoints lie in $G$. For each $n$, the set

$$
H_{n} \equiv\left(-\infty, a_{n}\right] \cup\left[b_{n},+\infty\right)
$$

is called a notched line. Clearly, each set $H_{n}$ is located and for any $x \in R$,

$$
\rho\left(x, H_{n}\right)=\left(\left(x-a_{n}\right) \wedge\left(b_{n}-x\right)\right) \vee 0 .
$$

For each $m$, the set

$$
G_{m} \equiv \bigcap_{n=1}^{m} H_{n}
$$

is called a finitely notched line. The theorem expresses a located set as an infinitely notched line.

(ii) Condition (1) is satisfied.

For any $n$, if $\left(a_{n}, b_{n}\right)$ is fixed, then $a_{n}<b_{n}$, while if $\left(a_{n}, b_{n}\right)$ is void, then redefine $a_{n} \equiv 1$ and $b_{n} \equiv 0$.

(iii) Condition (2) is satisfied.

Either $b_{n}<b_{k}$ or $b_{n}>a_{k}$. It follows from the disjointness of the intervals that in the first case $b_{n} \leqq a_{k}$ and in the second case $b_{k} \leqq a_{n}$. 
(iv) Condition (4) is satisfied, and thus

$$
G=\bigcap_{n} H_{n}=\bigcap_{m} G_{m} .
$$

Since $-H_{n} \cong-G$ it follows that $G \subseteq H_{n}$ for all $n$. Now let $x \in \bigcap_{n} H_{n}$. From $x \in-G$ it would follow that $x \in-H_{n}$ for some $n$; hence $x \in G$.

(v) Each set $G_{m}$ is located, with

$$
\rho\left(x, G_{m}\right)=\bigvee_{n=1}^{m} \rho\left(x, H_{n}\right)
$$

for all $x \in R$.

Let $\rho$ be the indicated maximum. Clearly $\rho$ is a lower bound for the set of numbers $|x-y|$, with $y \in G_{m}$. To show that $\rho$ is the infimum of this set it must be shown that whenever $\rho<\sigma$ there exists $y \in G_{m}$ such that $|x-y|<\sigma$. Equivalently, when $\rho<+\infty$ and $\varepsilon>0$ we must construct $y \in G_{m}$ such that $|x-y|<$ $\rho+\varepsilon$. Either $\rho(x, G)<\varepsilon$ or $\rho(x, G)>0$. In the first case construct $y \in G$ so that $|x-y|<\varepsilon$. In the second case choose $n$ so that $a_{n}<$ $x<b_{n}$. In the subcase $n>m$, put $y \equiv x$. In the subcase $n \leqq m$, then $\rho(x, G)=\rho\left(x, H_{n}\right)=\rho$; construct $y \in G$ such that $|x-y|<p+\varepsilon$.

(vi) For any finite interval $J$ and any $\varepsilon>0$ there exists $m$ such that

$$
\left|\rho(x, G)-\rho\left(x, G_{m}\right)\right|<\varepsilon
$$

for all $x$ in J. In this way a located set is approximated by finitely notched lines.

First consider the case in which $J$ consists of a single point $x$. Either $\rho(x, G)<\varepsilon$ or $\rho(x, G)>0$. In the first case $\rho\left(x, G_{1}\right)<\varepsilon$ because $G \leqq G_{1}$, so it suffices to put $m \equiv 1$. In the second case choose $m$ so that $x \in\left(a_{m}, b_{m}\right)$. Then $\rho(x, G)=\rho\left(x, G_{m}\right)$. Thus, in either case, $\left|\rho(x, G)-\rho\left(x, G_{m}\right)\right|<\varepsilon$. Note that although in general extended real numbers $s$ and $t$ can not be subtracted, even when $s \leqq t$, the indicated difference here is meaningful because the numbers involved are either finite or equal.

We may assume $J$ is fixed, and construct a finite $\varepsilon / 3$ approximation $A$ to $J$. For each $a$ in $A$ construct $m_{a}$ so that $\mid \rho(a, G)-$ $\rho\left(a, G_{m_{a}}\right) \mid<\varepsilon / 3$. Put $m \equiv \max \left\{m_{a}: a \in A\right\}$. To show that the finitely notched set $G_{m}$ approximates $G$ to within $\varepsilon$ on $J$, consider any point $x$ in $J$ and construct $a$ in $A$ such that $|x-a|<\varepsilon / 3$. It suffices to consider the second of the cases $\rho(x, G)<\varepsilon$ and $\rho(x, G)>2 \varepsilon / 3$. Choose $n$ so that $x \in\left(a_{n}, b_{n}\right)$. Then also $a \in\left(a_{n}, b_{n}\right)$ and $\rho(a, G)>\varepsilon / 3$; hence $\rho\left(a, G_{m_{a}}\right)>0$. Thus $n \leqq m_{a}$, so $n \leqq m$ and $\rho(x, G)=\rho\left(x, G_{m}\right)$.

(vii) The locating condition (3) obtains. 
Consider any $k$ and choose $M_{k}$ so that

$$
\left|\rho(x, G)-\rho\left(x, G_{M_{k}}\right)\right|<1 / 2 k
$$

for all $x$ in $(-k-1, k+1)$. Let $\left(a_{n}, b_{n}\right)$ meet $(-k, k)$ with $b_{n}-a_{n}>$ $1 / k$. Since the length of the interval $I \equiv\left(a_{n}, b_{n}\right) \cap(-k-1, k+1)$ is greater than $1 / k$, the midpoint $x$ of $I$ has a distance $\rho(x, G)>$ $1 / 2 k$. It follows that $n \leqq M_{k}$.

Sufficiency. Now let the sequences $\left\{a_{n}\right\}$ and $\left\{b_{n}\right\}$ satisfy conditions (1), (2) and (3), and let $G$ be defined by (4).

(viii) Definitions. Define the sets $H_{n}$ and $G_{m}$ as in (i) above. It follows that $G \equiv \bigcap_{n} H_{n}=\bigcap_{m} G_{m}$ and that all finite endpoints lie in $G$.

(ix) The set $G$ is closed.

Let $\left\{x_{k}\right\}$ be a sequence in $G$ with $x_{k} \rightarrow x$. To show $x \in G$, it suffices, because of condition (1), to consider a fixed notch $\left(a_{n}, b_{n}\right)$. It suffices to consider the first of the cases $x<b_{n}$ and $x>a_{n}$. Then $x_{k}<b_{n}$ eventually; it follows that $x_{k} \leqq a_{n}$ eventually, and thus $x \leqq$ $a_{n}$. Thus $x \in G$.

(x) For any $m$, the indexing of the $q$ fixed notches (if any) among the first $m$ may be rearranged so that

$$
a_{1}<b_{1} \leqq a_{2}<b_{2} \leqq \cdots \leqq a_{q}<b_{q} .
$$

We may assume that $q \geqq 2$ and that the intervals $\left(a_{n}, b_{n}\right)$ with $n \leqq q$ are the fixed intervals; let $b$ be the minimum of their right endpoints $b_{n}$. It follows that $b$ is finite. Choose $\varepsilon>0$ so that $\varepsilon<b_{n}-a_{n}$ for all $n \leqq q$. Since some $b_{n}$ is less than $b+\varepsilon$, we may by reindexing assume $b_{1}<b+\varepsilon$. It follows from condition (2) that $a_{1}<b_{1} \leqq a_{n}$ for $2 \leqq n \leqq q$. An induction completes the proof.

(xi) If $a \in R^{\infty}$ and $\varepsilon>0$, then for any $x \in R$ either $x>a$ or $x<a+\varepsilon$.

Of the cases $a<x$ and $a>-\infty$ we need consider only the second. Of the subcases $a<+\infty$ and $a>x$ we need consider only the first, and now $a$ is finite.

(xii) Consider any $m$ and the rearrangement of $(\mathrm{x})$, with $q \geqq 1$. For any $x \in R$ and any $\varepsilon>0$, one of the following holds

(a) $x<a_{1}+\varepsilon$.

(b) $a_{n}<x<b_{n}$ for some $n \leqq q$.

(c) $b_{n}-\varepsilon<x<a_{n+1}+\varepsilon$ for some $n<q$.

(d) $b_{q}-\varepsilon<x$.

By (xi), either $x<a_{1}+\varepsilon$ or $a_{1}<x$. An induction completes the proof.

(xiii) Each set $G_{m}$ is located, with 


$$
\rho\left(x, G_{m}\right)=\bigvee_{n=1}^{m} \rho\left(x, H_{n}\right)
$$

for all $x$ in $R$.

Let $\rho$ be the indicated maximum. For any $y \in G_{m}$, clearly $\rho \leqq$ $|x-y|$. Now let $\rho<+\infty$ and $\varepsilon>0$; we must construct $y \in G_{m}$ so that $|x-y|<\rho+\varepsilon$. Either $\rho>0$ or $\rho<\varepsilon$. In the first case choose $n \leqq m$ so that $\rho\left(x, H_{n}\right)>0$. Since $\rho\left(x, H_{n}\right)<\rho+\varepsilon$ one of the endpoints of $\left(a_{n}, b_{n}\right)$ is finite and suffices for $y$. In the second case, we may assume $q \geqq 1$ in (x), and according to the alternatives in (xii), define $y$ as follows.

(a) $y \equiv x \wedge a_{1}$.

(b) $y \equiv a_{n}$ or $y \equiv b_{n}$, as suitable.

(c) $y \equiv b_{n} \vee x \wedge a_{n+1}$.

(d) $y \equiv x \vee b_{q}$.

(xiv) Let $A$ be a set of extended real numbers. If for every $\varepsilon>0$ there exists $t$ in $A$ such that $t+\varepsilon$ is an upper bound for $A$, then $A$ has a supremum in $R^{\infty}$.

For each $n$, construct $t_{n}$ in $A$ such that $a \leqq t_{n}+1 / n$ for all $a$ in $A$. To show that $\left\{t_{n}\right\}$ converges in $R^{\infty}$ we apply [4, Theorem 3]. First we construct the auxiliary sequence $\left\{\sigma_{n}\right\}$. Note that if some $t_{n}<+\infty$ then clearly all $t_{n}<+\infty$. Thus we construct an increasing sequence $\left\{\sigma_{n}\right\}$ of 0 's and 1 's such that $t_{n}>n+1$ when $\sigma_{n}=0$ and $t_{n}<+\infty$ when $\sigma_{n}=1$. Now consider any $n$. If $\sigma_{n}=0$ then $n+1<t_{n} \leqq t_{k}+1 / k$ and thus $t_{k}>n$ for all $k$. If $\sigma_{n}=1$ and $k \geqq n$ then $t_{k} \leqq t_{n}+1 / n$ and $t_{n} \leqq t_{k}+1 / k$; thus $\left|t_{k}-t_{n}\right| \leqq 1 / n$. Thus we define $s \equiv \lim t_{n}$ and it follows that $s=\sup A$.

(xv) The set $G$ is located, with

$$
\rho(x, G)=\bigvee_{n} \rho\left(x, H_{n}\right)
$$

for all $x \in R$.

We first show that for any $x \in R$ the indicated supremum exists in $R^{\infty}$. By (xiii) it suffices to show that the distances $\rho\left(x, G_{n}\right)$ have a supremum. To verify the condition of (xiv), let $\varepsilon>0$ and choose $k$ so that $x \in(-k, k)$ and $1 / k<\varepsilon$. Using the locating parameter $M_{k}$ from condition (3), put $t \equiv \rho\left(x, G_{M_{k}}\right)$. We must show that $\rho\left(x, G_{n}\right) \leqq$ $t+\varepsilon$ for all $n$. For this it suffices to show $\rho\left(x, H_{n}\right) \leqq t+\varepsilon$ for all $n$. Consider any $n$. It suffices to consider the second of the cases $\rho\left(x, H_{n}\right)<\varepsilon$ and $\rho\left(x, H_{n}\right)>\varepsilon / 2$. Then $x \in\left(a_{n}, b_{n}\right)$ and $b_{n}-a_{n}>\varepsilon$. It follows from the locating condition (3) that $n \leqq M_{k}$, and thus $\rho\left(x, H_{n}\right) \leqq t$.

Thus the supremum exists; denote it by $\rho$. To show that $\rho \leqq|x-y|$ for every $y \in G$, let $\varepsilon>0$; we must show $\rho<|x-y|+$ $\varepsilon$. It suffices to consider the second of the cases $\rho<\varepsilon$ and $\rho>0$. 
Choose $m$ so that $\rho\left(x, H_{m}\right)>0$. Then $\rho=\rho\left(x, H_{m}\right) \leqq|x-y|$.

Now let $\rho<+\infty$ and $\varepsilon>0$. We must construct $y \in G$ such that $|x-y| \leqq \rho+\varepsilon$. Either $\rho>0$ or $\rho<\varepsilon$. In the first case choose $m$ so that $\rho\left(x, H_{m}\right)>0$. Since $\rho\left(x, H_{m}\right)<\rho+\varepsilon$, we obtain $|x-y|<\rho+\varepsilon$, where $y$ is a suitably chosen endpoint of $\left(a_{m}, b_{m}\right)$, and thus $y \in G$.

In the second case, where $\rho<\varepsilon$, we shall construct a point $y$ in $G$ such that $|x-y| \leqq \varepsilon$. First construct an increasing sequence $\left\{\tau_{k}\right\}$ of 0 's, and 1's, with $\tau_{1} \equiv 0$, such that $\rho<\varepsilon / k$ when $\tau_{k}=0$ and $\rho>0$ when $\tau_{k}=1$. Now construct a sequence $\left\{y_{k}\right\}$ of real numbers as follows. Consider any $k$. In the case $\tau_{k}=0$, we have $\rho\left(x, G_{k}\right)<$ $\varepsilon / k$; construct $y_{k} \in G_{k}$ such that $\left|x-y_{k}\right|<\varepsilon / k$. In the case $\tau_{k}=1$ first consider the subcase in which $k$ is the least integer, denoted $j$, of such integers $k$. Choose $n$ so that $a_{n}<x<b_{n}$. Since $\tau_{j-1}=0$ we have $\rho<\varepsilon /(j-1)$. Put $y_{j} \equiv a_{n}$ or $y_{j} \equiv b_{n}$ so that $\left|x-y_{j}\right|<$ $\varepsilon /(j-1)$. Since then $y_{j}$ is a finite endpoint, $y_{j} \in G$. When $k>j$ put $y_{k} \equiv y_{j}$. This defines the sequence $\left\{y_{k}\right\}$ with $\left|x-y_{k}\right|<\varepsilon$ and $y_{k} \in G_{k}$ for each $k$.

To show that $\left\{y_{k}\right\}$ is a Cauchy sequence, let $n>k$. Three cases result, depending on the values of $\tau_{k}$ and $\tau_{n}$. First, when both are 0 , we have $\left|x-y_{n}\right|<\varepsilon / n$ and $\left|x-y_{k}\right|<\varepsilon / k$, and thus $\left|y_{n}-y_{k}\right|<$ $2 \varepsilon / k$. Second, when both are 1 , then $y_{n}=y_{k}$. Finally, when $\tau_{k}=0$ and $\tau_{n}=1$, then $\left|x-y_{k}\right|<\varepsilon / k$ and $\left|x-y_{n}\right|<\varepsilon /(j-1) \leqq \varepsilon / k$. Thus $\left|y_{n}-y_{k}\right|<2 \varepsilon / k$. Hence $\left\{y_{k}\right\}$ is a Cauchy sequence converging to a point $y$ with $|x-y| \leqq \varepsilon$. From (ix) it follows that the sets $G_{k}$ are closed; thus $y \in G$.

Corollary. A countable union $\mathrm{U}_{n} I_{n}$ of fixative disjoint open intervals is colocated if and only if there exists a sequence $\left\{M_{k}\right\}$ of positive integers such that $n \leqq M_{k}$ whenever $I_{n}$ meets $(-k, k)$ and $\ell\left(I_{n}\right)>1 / k$.

Some applications of the theorem will be given in [5], where those located sets which have suprema in $R^{\infty}$, and those which are fixed, are characterized by means of numerical conditions on the endpoints of the notches.

\section{REFERENCES}

1. E. Bishop, Foundations of constructive analysis, McGraw-Hill, New York, 1967. MR 36 \#4930.

2. L. E.J. Brouwer, Begründung der Mengenlehre Unabhängig vom Logischen Satz vom Ausgeschlossenen Dritte II: Theorie der Punktmengen, Ver. Kon. Akad. Wet. (Amsterdam) 12 (1919), nr. 7.

3. M. Mandelkern, Components of an open set, to appear. 
4. M. Mandelkern, Resolutions on the line, Pacific J. Math., 88 (1980), 91-100.

5. - Suprema of located sets, J. London Math. Soc. (2), 20 (1979), 161-164.

Received May 4, 1979 and in revised form June 9, 1980.

New Mexico State University

Las CRUCes, NM 88003 



\section{PACIFIC JOURNAL OF MATHEMATICS}

\section{EDITORS}

DONALD BABBITT (Managing Editor)

University of California

Los Angeles, CA 90024

HUGo RossI

University of Utah

Salt Lake City, UT 84112

C. C. MOORE and ANDREW OGG

University of California

Berkeley, CA 94720
J. DugundjI

Department of Mathematics

University of Southern California

Los Angeles, CA 90007

R. FinN and J. Milgram

Stanford University

Stanford, CA 94305

\section{ASSOCIATE EDITORS}
R. ARENS
E. F. BECKENBACH
B. H. NeumanN
F. WOLF
K. YOSHIDA

\section{SUPPORTING INSTITUTIONS}

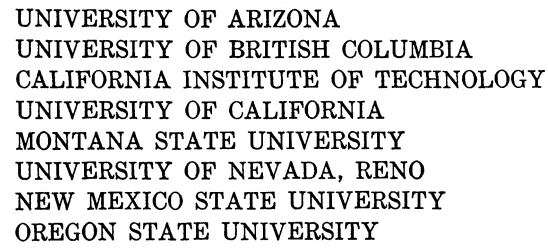

UNIVERSITY OF ARIZONA

UNIVERSITY OF BRITISH COLUMBIA

CALIFORNIA INSTITUTE OF TECHNOLOGY

UNIVERSITY OF CALIFORNIA

MONTANA STATE UNIVERSITY

UNIVERSITY OF NEVADA, RENO

NEW MEXICO STATE UNIVERSITY

OREGON STATE UNIVERSITY

\author{
UNIVERSITY OF OREGON \\ UNIVERSITY OF SOUTHERN CALIFORNIA \\ STANFORD UNIVERSITY \\ UNIVERSITY OF HAWAII \\ UNIVERSITY OF TOKYO \\ UNIVERSITY OF UTAH \\ WASHINGTON STATE UNIVERSITY \\ UNIVERSITY OF WASHINGTON
}

The Supporting Institutions listed above contribute to the cost of publication of this Journal, but they are not owners or publishers and have no responsibility for its content or policies.

Mathematical papers intended for publication in the Pacific Journal of Mathematics should be in typed form or offset-reproduced, (not dittoed), double spaced with large margins. Please do not use built up fractions in the text of the manuscript. However, you may use them in the displayed equations. Underline Greek letters in red, German in green, and script in blue. The first paragraph or two must be capable of being used separately as a synopsis of the entire paper. Please propose a heading for the odd numbered pages of less than 35 characters. Manuscripts, in triplicate, may be sent to any one of the editors. Please classify according to the scheme of Math. Reviews, Index to Vol. 39. Supply name and address of author to whom proofs should be sent. All other communications should be addressed to the managing editor, or Elaine Barth, University of California, Los Angeles, California, 90024.

50 reprints to each author are provided free for each article, only if page charges have been substantially paid. Additional copies may be obtained at cost in multiples of 50 .

The Pacific Journal of Mathematics is issued monthly as of January 1966. Regular subscription rate: $\$ 102.00$ a year (6 Vols., 12 issues). Special rate: $\$ 51.00$ a year to individual members of supporting institutions.

Subscriptions, orders for numbers issued in the last three calendar years, and changes of address shoud be sent to Pacific Journal of Mathematics, P.O. Box 969, Carmel Valley, CA 93924, U.S.A. Old back numbers obtainable from Kraus Per!odicals Co., Route 100, Millwood, NY 10546.

PUBLISHED BY PACIFIC JOURNAL OF MATHEMATICS, A NON-PROFIT CORPORATION

Printed at Kokusai Bunken Insatsusha (International Academic Printing Co., Ltd.). 8-8, 3-chome, Takadanobaba, Shinjuku-ku, Tokyo 160, Japan. 


\section{Pacific Journal of Mathematics}

\section{Vol. 95, No. $2 \quad$ October, 1981}

George E. Andrews, The Rogers-Ramanujan reciprocal and Minc's

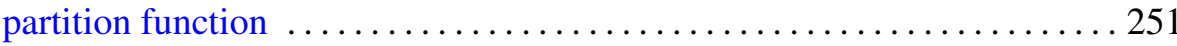

Allan Calder, William H. Julian, Ray Mines, III and Fred Richman,

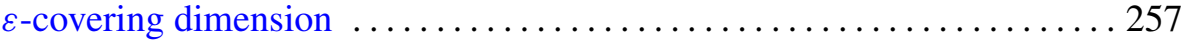

Thomas Curtis Craven and George Leslie Csordas, An inequality for the distribution of zeros of polynomials and entire functions $\ldots \ldots \ldots \ldots 263$

Thomas Jones Enright and R. Parthasarathy, The transfer of invariant

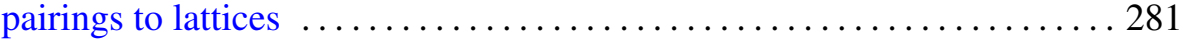

Allen Roy Freedman and John Joseph Sember, Densities and

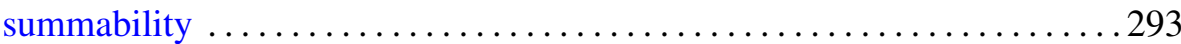

Robert Heller and Francis Aubra Roach, A generalization of a classical necessary condition for convergence of continued fractions . . . . . . 307

Peter Wilcox Jones, Ratios of interpolating Blaschke products ........... 311

V. J. Joseph, Smooth actions of the circle group on exotic spheres ........ 323

Mohd Saeed Khan, Common fixed point theorems for multivalued

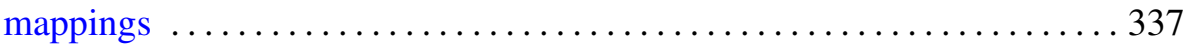

Samuel James Lomonaco, Jr., The homotopy groups of knots. I. How to

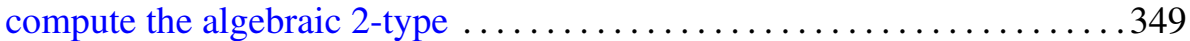

Louis Magnin, Some remarks about $C^{\infty}$ vectors in representations of connected locally compact groups ............................ 391

Mark Mandelker, Located sets on the line . . . . . . . . . . . . . . . . . 401

Murray Angus Marshall and Joseph Lewis Yucas, Linked quaternionic mappings and their associated Witt rings $\ldots \ldots \ldots \ldots \ldots \ldots \ldots \ldots . \ldots \ldots 11$

William Lindall Paschke, $K$-theory for commutants in the Calkin algebra

W. J. Phillips, On the relation $P Q-Q P=-i I$ 435

Ellen Elizabeth Reed, A class of Wallman-type extension. 443

Sungwoo Suh, The space of real parts of algebras of Fourier transforms 461 Antonius Johannes Van Haagen, Finite signed measures on function

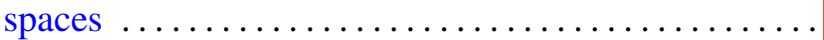

Richard Hawks Warren, Identification spaces and unique uniformity 